\title{
Alzheimer's disease: A review of its visual system neuropathology. Optical coherence tomography—a potential role as a study tool in vivo
}

\author{
J. P. Cunha ${ }^{1,2} \cdot$ N. Moura-Coelho ${ }^{1}$ - R. P. Proença ${ }^{1}$ - A. Dias-Santos ${ }^{1,2}$. \\ J. Ferreira ${ }^{1,2}$ - C. Louro $^{2}$ - A. Castanheira-Dinis ${ }^{3}$
}

Received: 25 April 2016/Revised: 12 June 2016 / Accepted: 22 June 2016

(C) Springer-Verlag Berlin Heidelberg 2016

\begin{abstract}
Alzheimer's disease (AD) is a prevalent, long-term progressive degenerative disorder with great social impact. It is currently thought that, in addition to neurodegeneration, vascular changes also play a role in the pathophysiology of the disease. Visual symptoms are frequent and are an early clinical manifestation; a number of psychophysiologic changes occur in visual function, including visual field defects, abnormal contrast sensitivity, abnormalities in color vision, depth perception deficits, and motion detection abnormalities. These visual changes were initially believed to be solely due to neurodegeneration in the posterior visual pathway. However, evidence from pathology studies in both animal models of AD and humans has demonstrated that neurodegeneration also takes place in the anterior visual pathway, with involvement of the retinal ganglion cells' (RGCs) dendrites, somata, and axons in the optic nerve. These studies additionally showed that patients with AD have changes in retinal and choroidal microvasculature. Pathology findings have been corroborated in in-vivo assessment of the retina and optic nerve head $(\mathrm{ONH})$, as well as the retinal and choroidal vasculature. Optical coherence tomography (OCT) in particular has shown great utility in the assessment of these changes, and it may become a useful tool for early detection and monitoring
\end{abstract}

J. P. Cunha and N. Moura-Coelho are both first authors.

J. P. Cunha

cunha.oft@gmail.com

1 CHCL - Centro Hospitalar de Lisboa Central, Lisbon, Portugal

2 Nova Medical School/Faculdade de Ciências Médicas (NMS/FCM), Universidade Nova de Lisboa, Centro Hospitalar de Lisboa Central, Alameda Santo António dos Capuchos, 1169-050 Lisbon, Portugal

3 VSRC-FML - Visual Sciences Research Centre, Faculty of Medicine of Lisbon, University of Lisbon, Lisbon, Portugal disease progression in $\mathrm{AD}$. The authors make a review of the current understanding of retinal and choroidal pathological changes in patients with $\mathrm{AD}$, with particular focus on invivo evidence of retinal and choroidal neurodegenerative and microvascular changes using OCT technology.

Keywords Alzheimer's disease · Optical coherence tomography $(\mathrm{OCT}) \cdot$ Retina $\cdot$ Choroid

\section{Introduction-Alzheimer's disease (AD)}

Alzheimer's disease $(\mathrm{AD})$ is a long-term progressive neurodegenerative disorder affecting the central nervous system (CNS). It is the most common cause of dementia, affecting about $6 \%$ of the population aged over 65 , and the clinical incidence doubles every 5 years after 65 years of age $[1,2]$. Over 26 million people are estimated to suffer from $\mathrm{AD}$, and this number is expected to quadruple by 2050 [3]. It is therefore a disease with a great social impact, leading to considerable impairment in quality of life for both patients, family members, and healthcare workers.

Its pathophysiology remains incompletely understood. The earliest $\mathrm{AD}$ pathological change in the CNS is the accumulation of amyloid $\beta(A \beta)$, derived from abnormal processing of amyloid precursor protein (APP). This process begins approximately 10 years before the onset of the clinical syndrome of dementia, which is characterized by a constellation of symptoms and signs manifested by difficulties in memory, disturbances in language, and psychological and psychiatric changes including behavioral disturbances, executive dysfunction, and impairment in activities of daily living [1,4]. Clinical progression shows great variability among patients.

Mild cognitive impairment (MCI) is an intermediate state of cognitive function between normal aging and early dementia, in which patients may have one or more cognitive domains 
affected, without impairment in activities of daily living. It is considered a risk factor for Alzheimer's disease, with variable progression to $\mathrm{AD}[5]$.

\section{Visual changes in AD patients}

Visual symptoms are frequently among the earliest symptoms in patients with Alzheimer's disease, and visual function is affected in most patients with $\mathrm{AD}$, contributing to further impairment in quality of life.

There are a number of changes in visual function in $\mathrm{AD}$ patients including visual field defects, predominantly in the inferior hemifield, abnormal contrast sensitivity, and abnormalities in color vision, depth perception, and visual sensitivity to motion [6]. Other psychophysiological changes such as visual acuity and pupillary changes have been studied in patients with $\mathrm{AD}$, with conflicting findings.

In addition, more complex visual symptoms may arise early in the history of AD, including visual memory deficits and visual hallucinations $[7,8]$.

However, the results of visual tests are dependent on the patient's understanding, memorization, vigilance, and compliance to the rules and the test instructions, which depends on the cognitive status of each patient. The ability to cooperate in some visual tests can be affected additionally by ocular motor abnormalities that may be present in patients with AD, such as slower saccadic movements and changes in smooth pursuit movement [9, 10]. Some of this data may thus be the result of poor compliance related to the cognitive status rather than the result dysfunction of the visual pathways [11].

\section{Neuropathology of the visual system in AD}

The two most important neuropathological features of AD are cerebral A $\beta$-amyloid neuritic plaques in neocortical terminal fields, and neurofibrillary tangles (NFTs) containing phosphorylated tau protein [12-14] initially in medial temporallobe structures and later in many forebrain and midbrain areas. Microvascular amyloid deposition (amyloid angiopathy), granulovacuolar degeneration, loss of neurons and white matter, synapse loss, gliosis, inflammation, and oxidative damage are other pathological changes present in $\mathrm{AD}$ [12-15].

\section{AD pathological changes in the posterior visual pathway}

Neurodegenerative changes have been demonstrated in most visual systems in AD. In fact, degenerative changes in the visual pathways occur early in the disease process of Alzheimer's, even before the onset of clinical symptoms of dementia $[15,16]$.

The visual cortex is relatively spared, and visual association areas (V2, V3) are more affected than primary visual cortex (area V1). In the primary visual cortex, the density of neuritic plaques and NFTs has been shown to be significantly greater in the cuneal gyrus compared to the lingual gyrus. This may account in part for the predominantly inferior VFDs in patients with AD [9].

Degeneration of lateral geniculate nucleus (LGN) has been documented in patients with AD [17]. Couser and Bernstein (2014) observed the presence of AD-related markers in the lateral geniculate nucleus (LGN), proposing that AD-related changes take place in this structure very early in the disease process [18].

Neurodegenerative changes also occur in the suprachiasmatic nucleus (SCN) [19]. This hypothalamic region is involved in sleep-wake circadian rhythm, receiving direct input from RGCs via the retino-hypothalamic tract. Degeneration of the SCN may account for the sleep disturbances found in patients with AD. Regarding sleep disturbances in $\mathrm{AD}$, it is of note that recent studies documented impairment of melanopsin RGCs (m-RGCs) in the retina of patients with AD. m-RGCs are a population of RGCs (1-2\% of total RGC population) involved in non-image-forming visual functions, including photoentrainment of circadian rhythms [20]. In this study, m-RGC density was significantly decreased in the AD group compared with controls, and remaining mRGCs presented impaired function, altered morphology, and amyloid deposits in and around these cells. These changes occurred in m-RGCs even with normal RGC counts, suggesting these cells may be specifically affected in AD. Further studies are necessary to understand whether trans-synaptic retrograde degeneration due to SCN pathology might or might not play an additional role in m-RGC loss in AD.

Oculomotor brain nuclei, namely the superior colliculus (SC), are also affected by AD-related neurodegenerative processes [21]. This may account, at least in part, for the abnormalities in eye movements previously described. There appears to be also amyloid plaque deposition and NFTs in pulvinar nuclei of patients with $\mathrm{AD}$, which may explain impaired visual attention in AD patients [22].

\section{Pathological changes in the retina and optic nerve}

Visual defects in AD patients were initially thought to be solely due to parietal and primary visual cortex pathology. However, increasing evidence has demonstrated that anterior visual pathway degeneration also plays a role. Hinton et al. first provided histopathological evidence of optic neuropathy and degeneration of retinal ganglion cells (RGC) in patients with AD, with a reduced number of RGCs and reduced retinal nerve fibre layer (RNFL) thickness [23]. Later, post-mortem studies showed that degeneration of the ganglion cell layer (GCL) occurs preferentially in superior and inferior quadrants, as well as in the central retina, in particular the temporal foveal region [24, 25]. Initially, a preferential loss of magnocellular 
RGC axons in the optic nerve was described [26], but other evidence suggests parvocellular RGC axon loss also takes place in AD [17]. Since then, pathology studies of AD patient's eyes have documented increased APP immunoreactivity, $A \beta$ and $p$ Tau deposits in the internal retinal layers (GCL, RNFL), retinal pigmentary epithelium, and retinal vasculature [27, 28], as well as reduction of choroidal thickness [29].

Animal models of AD have provided further insight regarding retinal changes in Alzheimer's disease. A study using mouse models of $\mathrm{AD}$ demonstrated that formation of neuritic plaques in the retina and choroidal vasculature occurs prior to their manifestation in the brain [27], and amyloid deposits were found in RNFL, RGCs, internal retinal layers, and even in the photoreceptor outer segments. Interestingly, this study also analyzed post-mortem human retinae of patients with $\mathrm{AD}$, and observed a relation between severity of dementia and retinal amyloid plaque burden.

Evidence has emerged that degeneration of RGC dendritic processes in the internal plexiform layer (IPL) may take place before RGC loss, suggesting synaptic dysfunction may be an early change in the neurodegenerative process [30]. In addition, activated glial cells, hypertrophy of retinal pigmented epithelial cells, and choroidal inflammation have also been observed in the eyes of mouse models of Alzheimer's disease [3].

These neuropathological findings have raised the possibility that the changes in the retinae of patients with $\mathrm{AD}$ may be due to both direct retinal pathology and trans-synaptic retrograde degeneration. Trans-synaptic retrograde degeneration (TRD) of neurons is a process occurring after central nervous system lesions, in which degeneration of the axon proceeds towards the cell body, as opposed to anterograde (Wallerian) degeneration. This creates a hostile, neurotoxic environment that leads to secondary degeneration of neighboring neurons. TRD has been demonstrated in a number of neurological pathways; interest in TRD in the visual pathway has arisen since Van Buren et al. (1963) first observed atrophy of the right LGN and retinal ganglion cells (RGCs) following right occipital lobectomy in an adolescent monkey [31]. Later, pathology studies in humans with occipital lesions also revealed changes in LGN [32]. Clinical evidence further supported this hypothesis. Fletcher et al. (1988) described optic disc pallor and band atrophy in individuals with congenital occipital lesions. Initially it was believed that TRD in the visual pathway occurred only in individuals with congenital (prenatal) occipital lobe lesions [33], although recent studies have also provided evidence that TRD of RGC can also occur in patients with acquired occipital lobe lesions [34-36].

\section{Imaging of retina, optic nerve, and choroid in AD}

In-vivo morphological studies have provided further evidence of both direct involvement of the retina and optic nerve head, and TRD processes in patients with $\mathrm{AD}$, as well as choroidal changes.

Initial in-vivo non-invasive studies of optical neuropathy in patients with Alzheimer's disease using fundus photographs showed retinal nerve fibre layer (RNFL) abnormalities, as well as macular changes and $\mathrm{ONH}$ abnormalities (increased cup-to-disc ratio and decreased neurorretinal rim) [37-39]. Increased cup-to-disc ratio has been reported to correlate with RNFL thinning in AD [40-42], and cup-to-disc ratio may be correlated with duration of disease [37]. This method, however, has certain disadvantages, as it is manual and highly subjective to each individual observer [41].

Later noninvasive studies of RNFL and ONH using scanning laser ophthalmoscopy (SLO) [42], and time-domain OCT (TD-OCT) studies confirmed the reduction in peripapillary RNFL thickness [40, 43-47]. In addition, invivo studies have shown reduced macular thickness and volume in patients with AD [44, 48-54].

Pathological changes in Alzheimer's disease not only involve neurodegeneration, but also the vasculature. The notion of $\mathrm{AD}$ as a disease of the neurovascular unit emerged from the findings of cerebral amyloid angiopathy, frequent focal changes in brain microcirculation, and especially because these vascular changes in AD are intimately associated with the typical neuronal degenerative changes [55]. Recent evidence has raised the possibility that the retinal microvasculature is affected in AD. Laser Doppler studies have shown altered retinal blood flow in patients with $\mathrm{AD}$, although no correlation was found between RNFL thickness and retinal blood flow [45]. Two recent studies using retinal photography found retinal vascular abnormalities, including vascular attenuation, reduced branching pattern, and altered venular tortuosity [54, $56,57]$. According to the authors, these retinal vascular changes in AD may be correlated with the neocortical amyloid plaque burden [58] or may mirror the cerebral microvascular pathology [54].

The choroid also appears to be affected in AD. Atrophy of the choriocapillaris has been shown to occur with normal aging, and age-dependent amyloid accumulation in the choroid vasculature has been demonstrated in mice and also in transgenic mouse models of AD [59].

\section{Optical coherence tomography—a potential new biomarker for detection of AD}

Early optical coherence tomography studies have shown peripapillary RNFL thinning in AD patients, although discrepancy has been found concerning which quadrants were most affected. More recent OCT studies have reported also macular changes in the ganglion cell complex (GCC), comprising the ganglion cell layer (GCL) and inner plexiform layer (IPL). Also, choroidal thickness [60] has been shown 

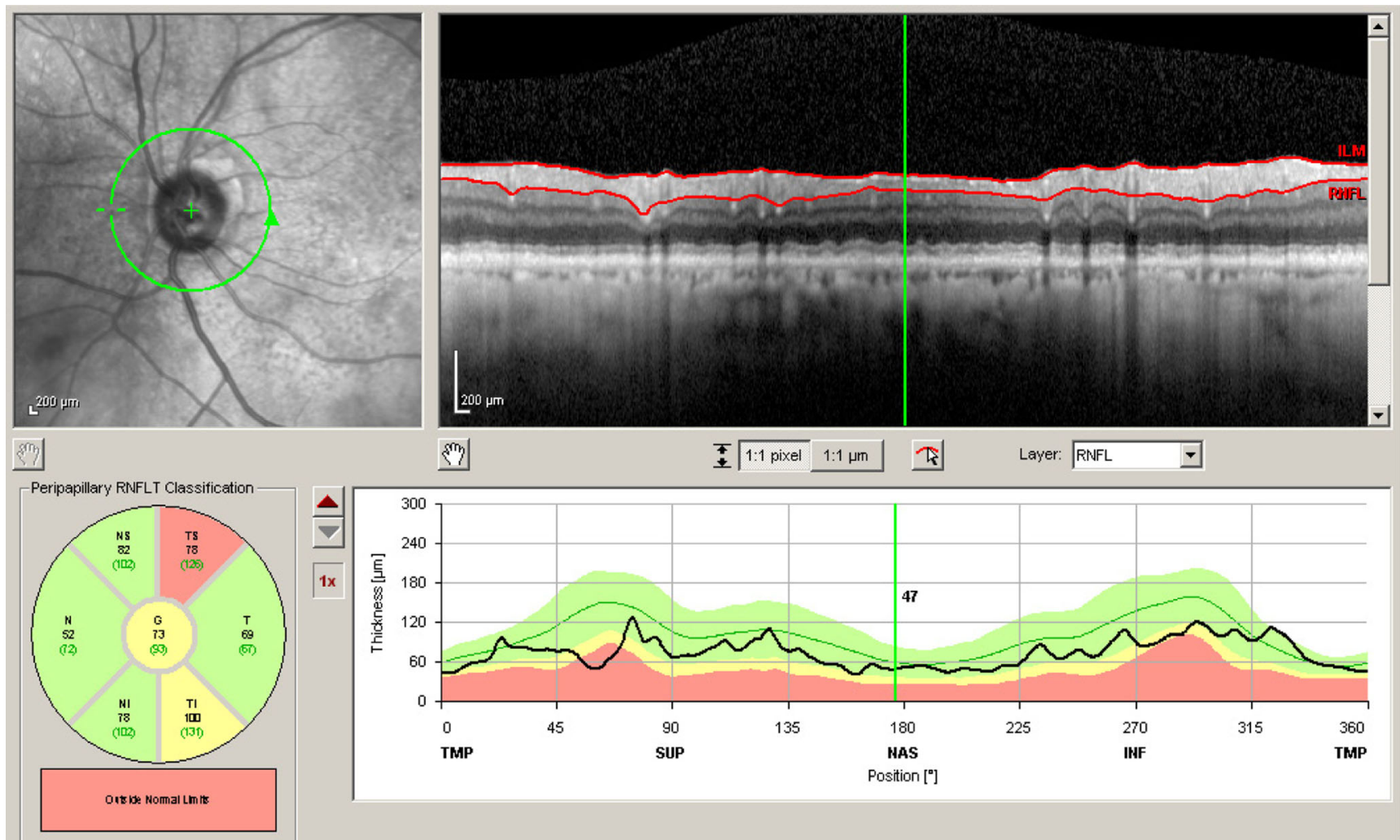

\section{smy}

I $1: 1$ pixel $1: 14 \mathrm{~mm}$ 요

Layer: RNNL

$\checkmark$

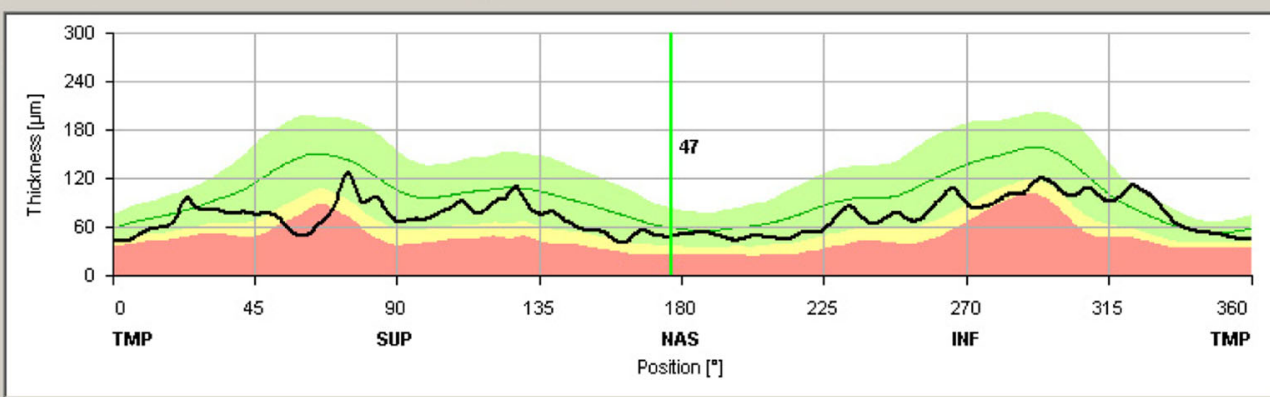

Fig. 1 Reduced RNFL thickness in Alzheimer's Disease, as measured by SD-OCT (Spectralis Heidelberg). In this case, diffuse RNFL thinning is documented, in particular due to thinning in the temporal superior and temporal inferior sectors

to be reduced in patients with $\mathrm{AD}$, as measured by EDI-OCT Spectralis Heidelberg [61-63].

The evaluation of visual changes in $\mathrm{AD}$ patients has been difficult, due to difficulties in patient collaboration, and limitations in diagnostic exams to overcome these difficulties. These factors have long interfered with the interpretation of findings in these patients.

Nevertheless, results from the studies of retinal and choroidal changes in $\mathrm{AD}$ patients have raised the possibility that
OCT may be a non-invasive clinical biomarker for the early detection of $\mathrm{AD}$. Retinal changes may be an early event in the course of $\mathrm{AD}$, and retinal $\mathrm{OCT}$ may provide insights for assessing neurodegeneration in the brain [50].

OCT is a reliable non-invasive, trans-pupillary technique that provides high-resolution cross-sectional images of RNFL and macular volume, allowing visualization of axons of the central nervous system (CNS) and evaluation of RNFL thickness [39, 41]. Peripapillary RNFL thickness (RNFLT) is

Fig. 2 Automatic segmentation of retinal layers in macular region as measured by SD-OCT (Spectralis Heidelberg)
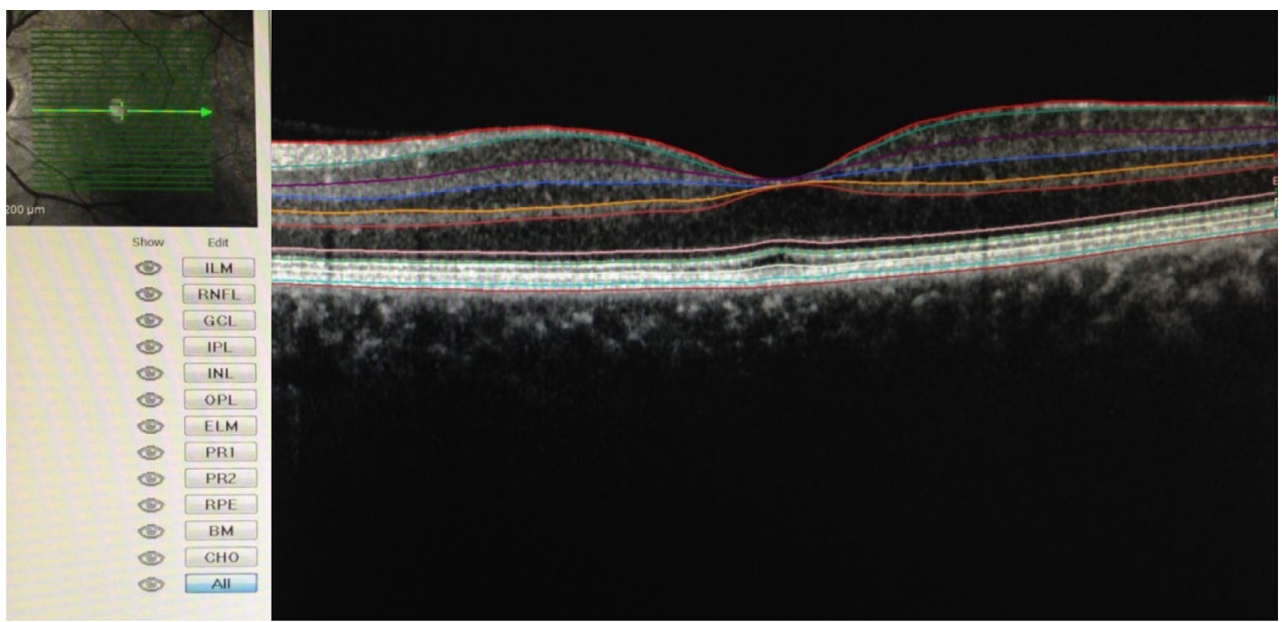


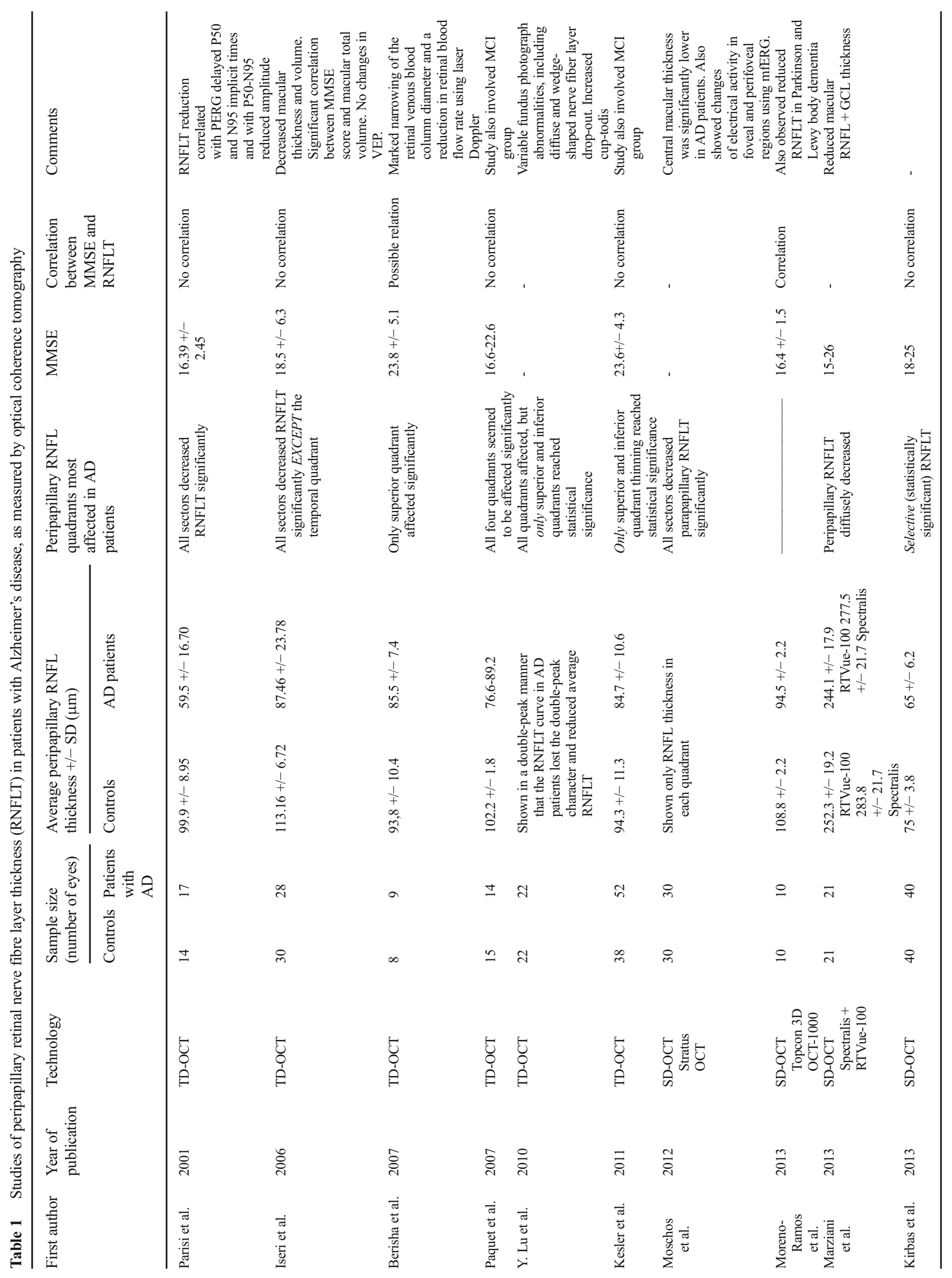




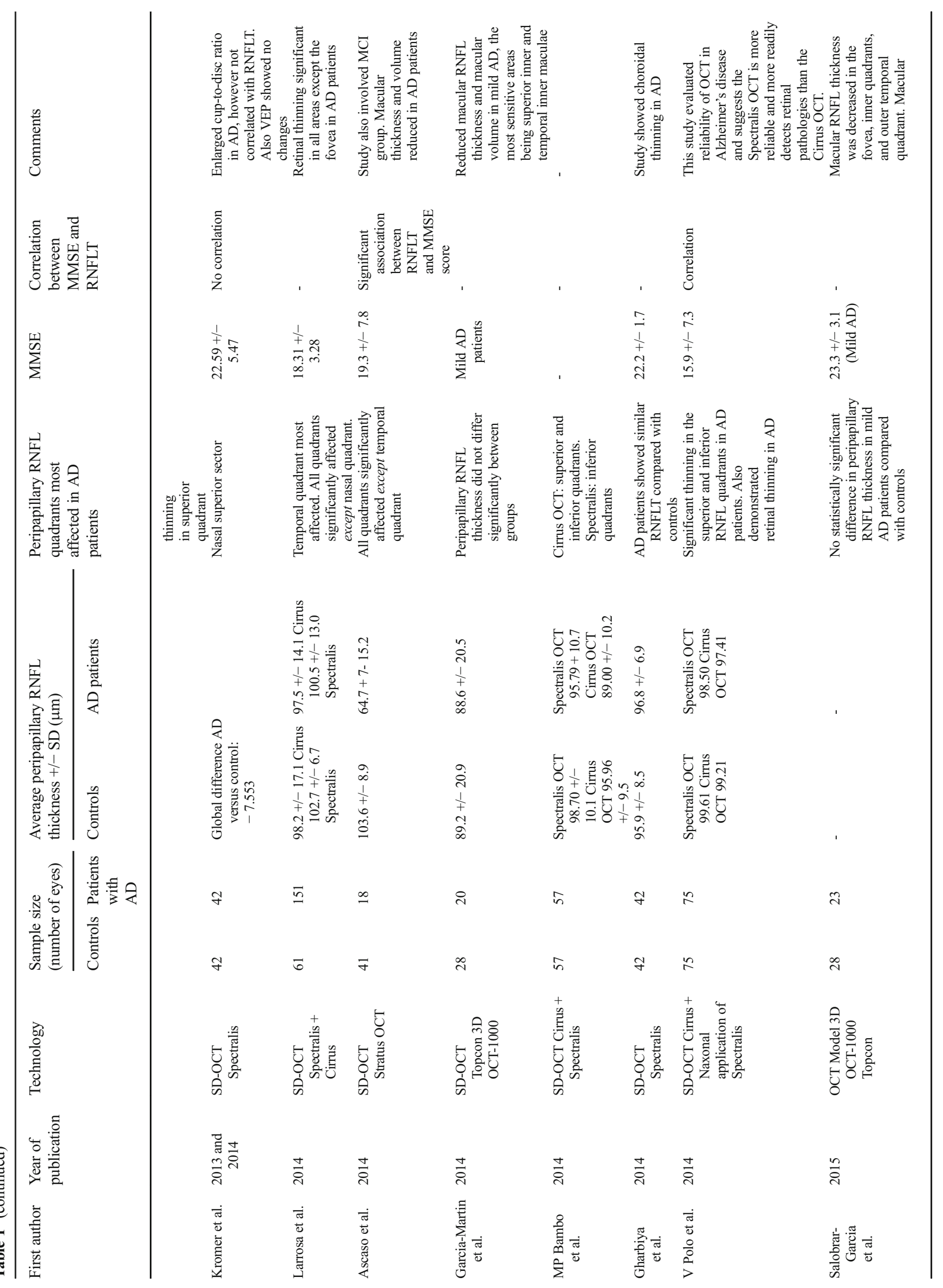




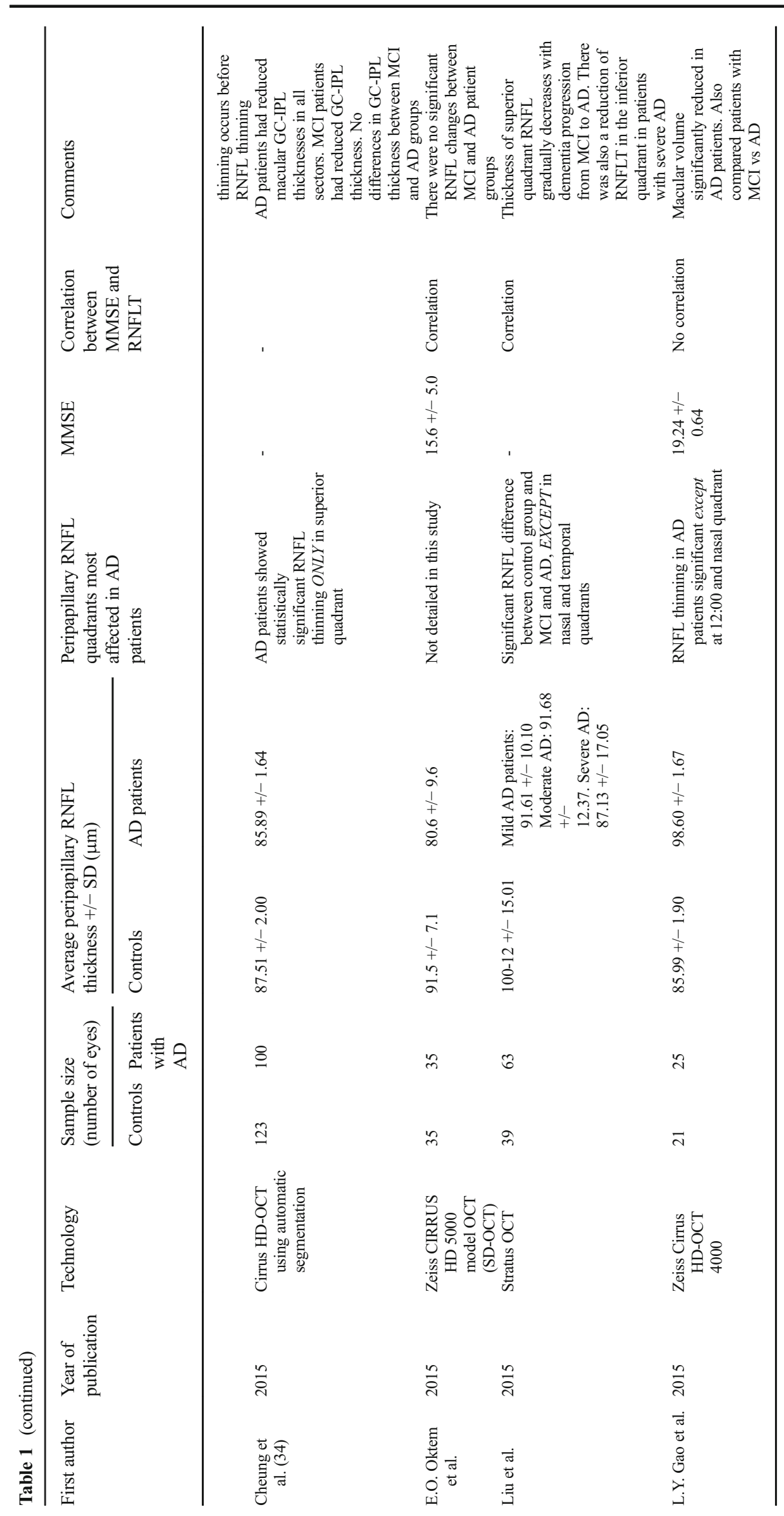




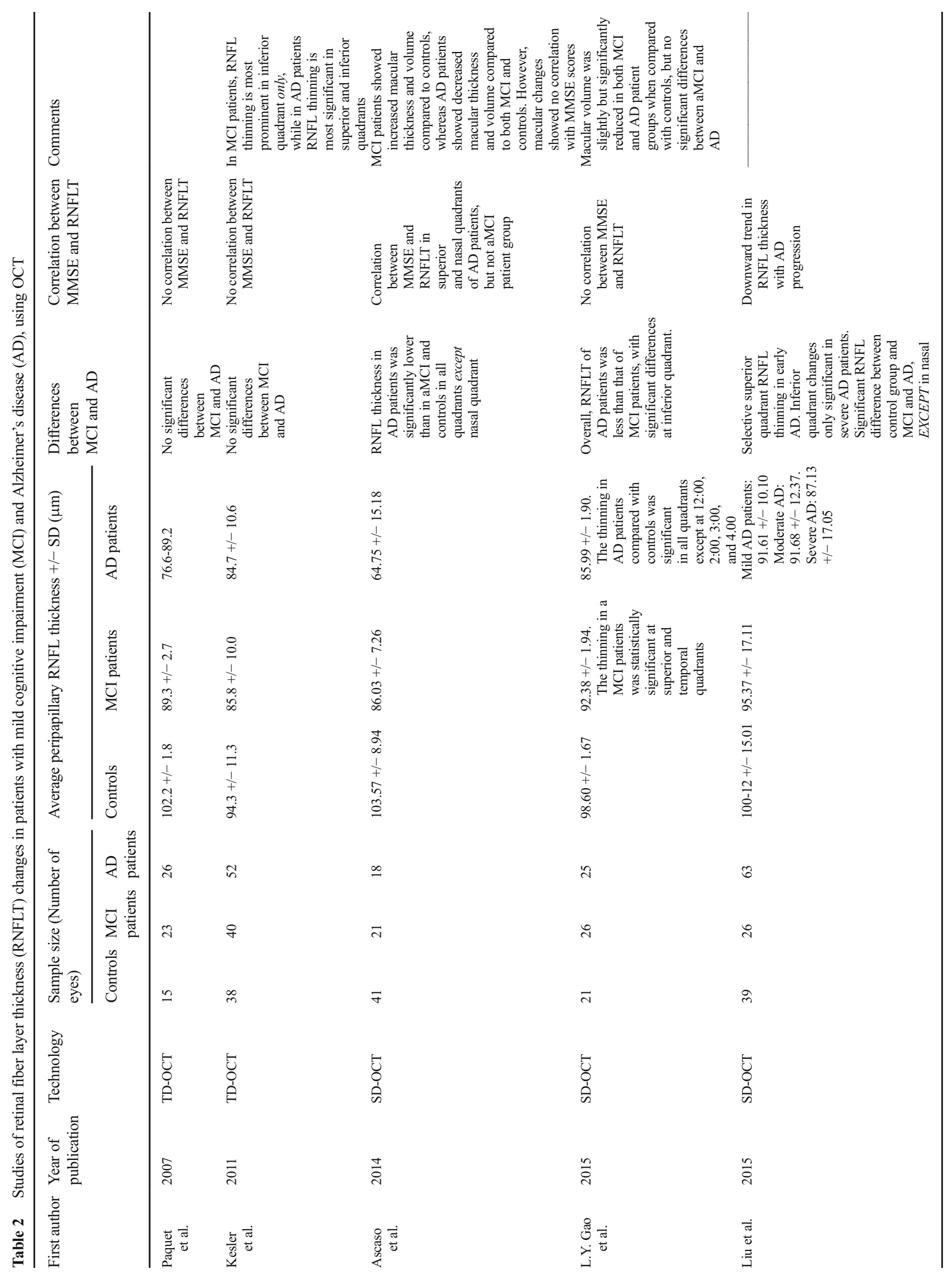


believed to reflect axonal loss, whereas macular volume reflects neuronal loss of RGC, since the neuronal bodies and dendrites are located in the ganglion cell/internal plexiform layer (GC-IPL), mostly in the macula lutea [50].

TD-OCT has been the most widely employed ocular imaging technique for assessment of optic nerve degeneration in $\mathrm{AD}$ [43-47], as well as for evaluating macular thinning in these patients [44].

Spectral-domain OCT (SD-OCT), a Fourier domain OCT technique, provided dramatically increased scanning speed and higher axial resolution when compared to TD-OCT technology, allowing the study of RGC and analysis of the GCIPL layers $[41,49]$. SD-OCT has been validated as a reliable technique for detecting subclinical RNFL and retinal atrophy in $\mathrm{AD}$ [64-66]. A number of studies using this technology have corroborated peripapillary RNFL thinning findings from TD-OCT studies. Figure 1 shows these changes in pRNFL thickness. In addition, one study using SD-OCT showed a diffuse reduction of the RNFL and GCL combined in AD [49], although the authors were not able to determinate which layer was most affected by AD. Later studies have demonstrated IPL thinning in AD patients [41, 50, 52, 56, 57]. This reduction of ganglion cell complex thickness (GC-IPL and RNFL layers) in AD occurs to a larger extent than that accounted for age-related GC-IPL loss alone (about $0.3 \mu \mathrm{m} /$ year) [54]. Macular GC-IPL thinning may be a more sensitive marker of earlier neurodegeneration in $\mathrm{MCI}$ and $\mathrm{AD}$ than evaluation of the retinal fibre layer. These findings are in agreement with the pathological findings of direct retinal involvement in dementia. Figure 2 illustrates the GC-IPL thinning as measured by SD-OCT.

Evidence from OCT studies also supports a role for TRD in disorders of the posterior visual pathway $[34,35,67,68]$ with visual field defects in these disorders correlating best with macular GCL thickness than with RNFL thickness $[67,68]$. Likewise, OCT findings in AD suggest the contribution of TRD in the pathophysiology of visual changes. In addition to previous OCT studies showing reduced peripapillary RNFL thickness, a recent SD-OCT study found that reduced grey matter volumes of occipital and temporal lobes was independently associated with thinning of the GC-IPL and peripapillary RNFL in individuals without dementia [69]. Since those cortical regions are an early site of deposition of senile plaques and NFTs, the findings by Y.-T. Ong and coworkers raises the possibility that GC-IPL thinning may reflect neurodegenerative changes in the brain, even before the clinical onset of dementia.

Some questions remain to be answered before considering OCT a useful clinical biomarker for early detection of dementia and assessment of disease progression in AD. As stated earlier, peripapillary RNFL thinning has been demonstrated in a number of studies. However, differences have been reported regarding which retinal quadrants are 
most affected. While some studies report preferential superior quadrant RNFL thinning in $\mathrm{AD}$, which would explain the predominantly inferior visual field defects previously described in $\mathrm{AD}[40,45,47,54,70-72]$, other authors have reported different findings; these results are presented in Table 1. A meta-analysis of TD-OCT studies in AD demonstrated that peripapillary RNFL thinning occurred in all retinal quadrants [73]. However, more recent SD-OCT studies have reported different findings, with most SD-OCT studies suggesting selective superior RNFL thinning [39, 54, 70]. Two more recent meta-analyses, which used both TD-OCT and SD-OCT studies, aimed to determine the diagnostic utility of OCT measurements of RNFL thickness in $\mathrm{AD}[74,75]$. While confirming significant peripapillary RNFL thinning in $\mathrm{AD}$, these meta-analyses reached different conclusions regarding which quadrants are most affected. Whether or not a correlation exists between retinal changes and severity of dementia also remains a controversial issue. While most OCT studies did not show correlation between Minimal Mental State Examination (MMSE) and peripapillary RNFL thinning (Table 2), one TD-OCT study reported correlation between MMSE scores and macular volume [44], and more recent studies using SD-OCT have indeed reported a significant correlation between MMSE scores and RNFL thickness [50, 66, 72, 76, 77]. Studies comparing changes in RNFLT in MCI and AD (Table 2) have also yielded conflicting results, although the majority reported no statistically significant differences between MCI and AD patient groups [46, 47, 54, 77]. The metaanalyses by K.L. Thomson et al. and G. Coppola et al. $[74,75]$ also tried to determine the utility of OCT as a tool for evaluating disease progression, and prognostic significance of GC-IPL and RNFL thickness in MCI and AD. Both meta-analyses concluded that OCT can be used to detect early abnormalities in MCI, but their conclusions regarding which retinal quadrants are affected in MCI were conflicting - one concluded that MCI patients have selective superior quadrant RNFL thinning [74], while the other concluded that all quadrants but the superior one were affected [75]. In addition, they failed to determine a correlation between RNFL and clinical severity of dementia [74].

Spectral-domain OCT (SD-OCT) using enhanced depth imaging modality (EDI) is a technology that allows visualization of the deeper structures of the eye such as the choroid. Choroidal thickness (CT) varies topographically within the posterior pole, being thickest under the fovea and thinnest in the nasal area. EDI-OCT has been validated as a reproducible modality for subfoveal CT measurements [60]. In addition, EDI-OCT findings have confirmed that CT significantly decreases with older age.

Choroidal thickness (CT) has been shown to be reduced in all regions in patients with $\mathrm{AD}$, as measured by EDI-OCT Spectralis Heidelberg [61]. Figure 3 depicts these changes in choroidal thickness. These findings have been corroborated by further studies [62, 63]. One study also included patients with MCI, and observed reduced CT in this group, although no statistically significant differences between the $\mathrm{MCI}$ and $\mathrm{AD}$ patient groups were found [63]. Although CT is reduced, it remains to be elucidated whether or not choroidal thinning is correlated with MMSE scores, as authors report conflicting results. The results of these studies are shown in Table 3.

Swept source-OCT (SS-OCT) is another Fourier domain OCT technology. It has also an improved signalto-noise ratio as compared to TD-OCT, providing higher image resolution. SS-OCT additionally offers some advantages over SD-OCT, including higher detection efficiency, increased sensitivity with image depth, reduced fringe washout and longer imaging range, higher penetration into the $\mathrm{ONH}$ and choroid, and lowered sensitivity to ocular opacities [41].

Ultrahigh-speed OCT based on swept laser technology enables rapid 3D imaging of the retina and Doppler OCT examination of ocular circulation with a non-invasive ocular angiography by optical coherence tomography. This can be a powerful methodology for measuring total retinal blood flow, which might be beneficial for assessing pathologic changes in diseases with impaired circulation of retina and choroid such as $\mathrm{AD}[78]$.

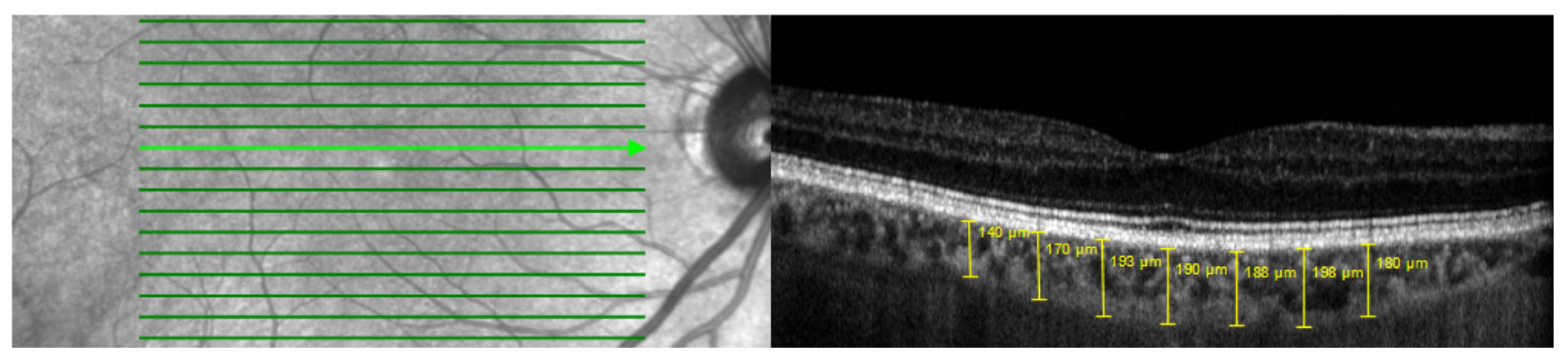

Fig. 3 Choroidal thickness $(C T)$ as measured by EDI-OCT (Spectralis Heidelberg) 


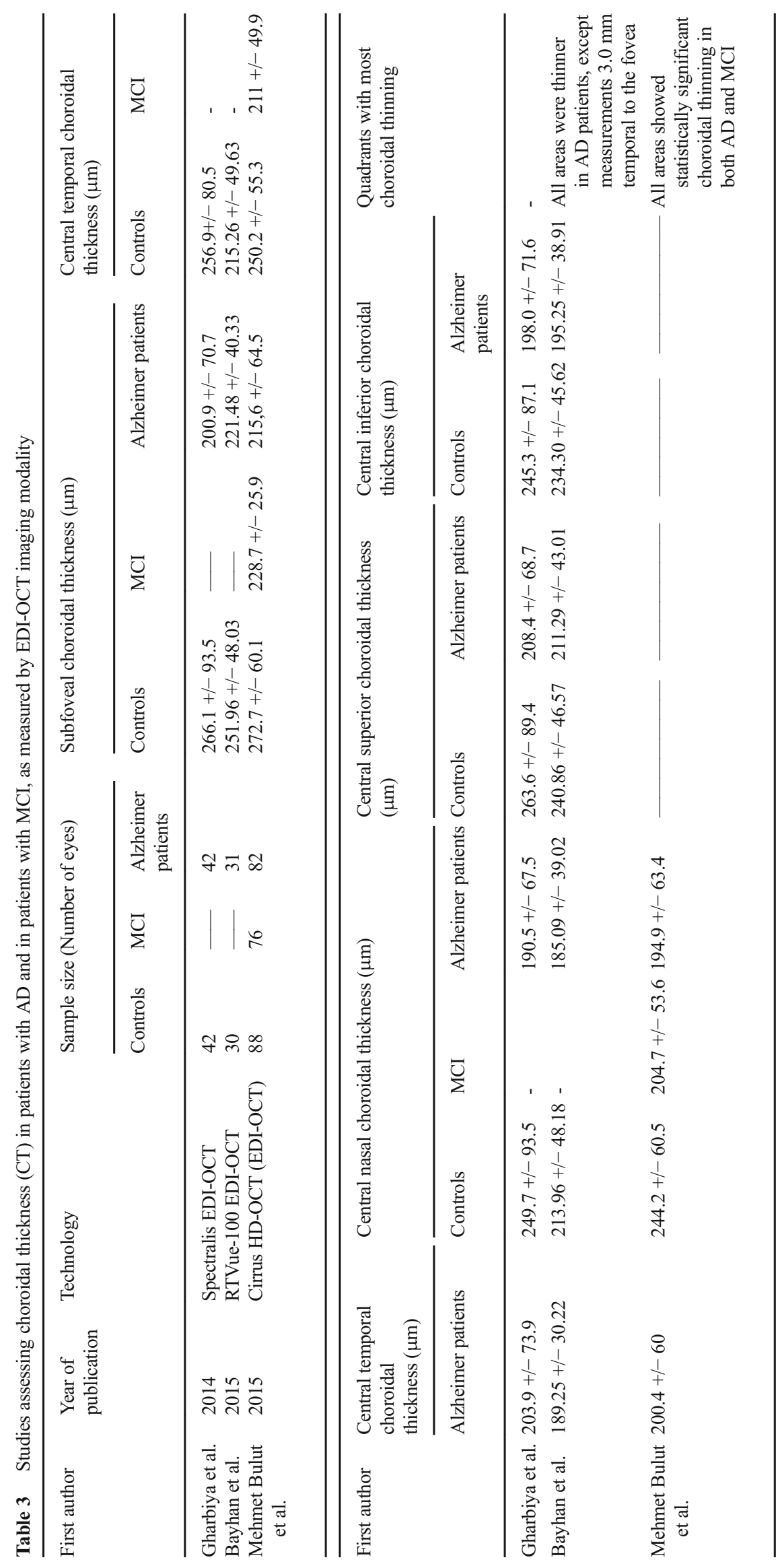




\section{Conclusions}

Patients with signs and symptoms of dementia undergo neuropsychological evaluation, and several clinical tools have been validated for that purpose, including the Clinical Dementia Rating (CDR) and Alzheimer's Disease Assessment Scale (ADAS). Diagnostic criteria can be used for clinical diagnosis of Alzheimer's disease, namely the National Institute of Neurological and Communicative Disorders and Stroke and the Alzheimer's Disease and Related Disorders Association (NINCDS-ADRDA) criteria [68] and the Diagnostic and Statistical Manual of Mental Disorders (DSM-V) criteria. However, to date, the definitive diagnosis of $\mathrm{AD}$ can still only be made on pathologic examination. However, a number of diagnostic exams have been considered to be supportive of the clinical diagnosis. Neuroimaging technologies, namely computerized tomography (CT) scanning or magnetic resonance imaging (MRI) have shown medial temporal lobe atrophy. Reduced brain activity has been detected by changes in glucose metabolism in positron emission tomography (FDG-PET). Biochemical cerebrospinal fluid (CSF) markers have been suggested as potential biomarkers for early detection of Alzheimer's disease (decreased levels of CSF $\mathrm{A} \beta_{42}$ protein and increased CSF levels of tau and phosphorylated tau proteins) $[79,80]$.

Cicero (106-43 BC) stated "the face is a picture of mind as the eyes are its interpreter"; Matthew reveals in the Bible "the eye is the lamp of the body", and it is attributed to Shakespeare the quote "the eyes are the window to the soul".

Optical coherence tomography findings in many neurodegenerative diseases have revived this notion of the eye as the "window to the brain". Anatomically and embryologically, the retina is known as an extension of the central nervous system; the fibers of retinal ganglion cell axons forming the optic nerve are the axons of the CNS and the retinal and cerebral vasculature share similar anatomic, physiological, and embryological characteristics.

OCT studies have provided useful information regarding retinal and choroidal changes in the eyes of patients with $\mathrm{AD}$, as OCT findings are consistent with the findings from pathology studies of both human and animal models of AD. In addition, retinal changes in microvasculature may reflect microvascular disease in the brain.

These findings may render OCT a useful clinical tool for diagnostic and prognostic evaluation of $\mathrm{MCI}$ and $\mathrm{AD}$ patients. Newer, more sensitive imaging modalities for measurements of retinal layers and choroidal thickness will allow further understanding of the earliest changes in the retina of these patients. It is likely that macular inner retinal layers (GC-IPL and macular RNFL) neurodegenerative changes are the earliest events, followed by peripapillary RNFL thinning, but vascular changes both in choroid and in retina may also play a role in the initiation of the neurodegenerative processes. Further OCT studies are thus needed to determine the progression of these neurovascular degenerative changes in MCI and AD.

\section{Literature research}

We searched the PubMed and Google Scholar databases to identify articles published up to 27th March 2016, using the following key terms: "Alzheimer's disease", "dementia", "optical coherence tomography", "visual function abnormalities". In addition, we manually searched the reference lists of most primary articles and reviewed articles.

\section{Compliance with ethical standards}

Funding No funding was received for this research.

Conflicts of interest All authors certify that they have no affiliations with or involvement in any organization or entity with any financial interest (such as honoraria; educational grants; participation in speakers' bureaus; membership, employment, consultancies, stock ownership, or other equity interest; and expert testimony or patent-licensing arrangements), or non-financial interest (such as personal or professional relationships, affiliations, knowledge or beliefs) in the subject matter or materials discussed in this manuscript.

Ethical approval All procedures performed in studies involving human participants were in accordance with the ethical standards of the institutional and/or national research committee and with the 1964 Helsinki declaration and its later amendments or comparable ethical standards.

Informed consent Informed consent was obtained from all individual participants included in the study, and for this type of study, formal consent is not required. This article does not contain any studies with human participants or animals performed by any of the authors.

\section{References}

1. Burns A et al (2009) Alzheimer's disease. BMJ 338:b158

2. Querfurth HW, Laferla FM (2010) Alzheimer's disease. N Engl J Med 362:329-344

3. Tsai Y, Lu B, Ljubimov AV et al (2014) Ocular changes in TgF344$\mathrm{AD}$ rat model of Alzheimer's disease. Invest Ophthalmol Vis Sci 55:523-534. doi:10.1167/iovs.13-12888

4. Bird TD, Miller BL (2008) Alzheimer's disease and primary dementias. In: Fauci AS, Braunwald E, Kasper DL, Hauser SL, Longo DL, Jameson JL, Loscalzo J (eds) Harrison's principles of internal medicine, 17th edn. McGraw-Hill, New York, NY, pp 2393-2406

5. Petersen RC (2011) Mild cognitive impairment. N Engl J Med 364: 2227-2234. doi:10.1056/NEJMcp0910237

6. Rizzo M, Nawrot M (1998) Perception of movement and shape in Alzheimer's disease. Brain 121(12):2259-2270

7. Murgatroyd C, Prettyman R (2001) An investigation of visual hallucinosis and visual sensory status in dementia. Int $\mathrm{J}$ Geriatr Psychiatry 16:709-713

8. Chapman FM, Dickinson J, McKeith I et al (1999) Association among visual hallucinations, visual acuity, and specific eye 
pathologies in Alzheimer's disease: treatment implications. Am J Psychiatry 156:1983-1985

9. Tzekov R, Mullan M (2013) Vision function abnormalities in Alzheimer disease. Surv Ophthalmol 59(4):414-433

10. Chang LYL, Lowe J, Ardiles A, Lim J, Grey AC (2014) Alzheimer's disease in the human eye. Clinical tests that identify ocular and visual information processing deficit as biomarkers. Alzheimers Dement 10:251-261

11. Drobny JV, Anstey KJ, Andrews S (2005) Visual memory testing in older adults with age-related visual decline: a measure of memory performance or visual functioning? J Clin Exp Neuropsychol 27: 425-435

12. Hyman BT et al (2012) National Institute on aging-Alzheimer's Association guidelines for the neuropathologic assessment of Alzheimer's disease. Alzheimers Dement 8:1-13. doi:10.1016/j. jalz.2011.10.007

13. Perl DP (2010) Neuropathology of Alzheimer's disease. Mt Sinai J Med 77(1):32-42

14. Sawa GM, Wharton SB, Lince PG, Forster G, Matthews FE, Brayne C (2009) Medical research council cognitive function and ageing study. Age, neuropathology, and dementia. N Engl J Med 360(2302):9

15. Parvizi J, Van Hoesen GW, Damasio A (2001) The selective vulnerability of brainstem nuclei to Alzheimer's disease. Ann Neurol 49:53-66

16. Katz B, Rimmer S (1989) Ophthalmologic manifestations of Alzheimer's disease. Surv Ophthalmol 34:31-43

17. Leuba G, Saini K (1995) Pathology of subcortical visual centres in relation to cortical degeneration in Alzheimer's disease. Neuropathol Appl Neurobiol 21:410-422

18. Couser E, Bernstein SL (2014) Alzheimer-induced changes in biomarkers in the human lateral geniculate nucleus. Invest Ophthalmol Vis Sci 55(13):1850-1850

19. Stopa EG, Volicer L, Kuo-Leblanc V et al (1999) Pathologic evaluation of the human suprachiasmatic nucleus in severe dementia. $\mathrm{J}$ Neuropathol Exp Neurol 58:29-39

20. La Morgia C, Ross-Cisneros FN, Sadun AA, Hannibal J, Munarini A, Mantovani V, ... \& Salomao SR (2010) Melanopsin retinal ganglion cells are resistant to neurodegeneration in mitochondrial optic neuropathies. Brain awq155

21. Iseki E, Matsushita M, Kosaka K et al (1989) Distribution and morphology of brain stem plaques in Alzheimer's disease. Acta Neuropathol 78:131-136

22. Kuljis RO (1994) Lesions in the pulvinar in patients with Alzheimer's disease. J Neuropathol Exp Neurol 53:202-211

23. Hinton DR, Sadun AA, Blanks JC, Miller CA (1986) Optic-nerve degeneration in Alzheimer's disease. N Engl J Med 315:485-487. doi:10.1056/NEJM198608213150804

24. Blanks JC, Torigoe Y, Hinton DR, Blanks RH (1996) Retinal pathology in Alzheimer's disease I. Ganglion cell loss in foveal/ parafoveal retina. Neurobiol Aging 17:377-384

25. Blanks JC, Schmidt SY, Torigoe Y et al (1996) Retinal pathology in Alzheimer's disease. II. Regional neuron loss and glial changes in GCL. Neurobiol Aging 17:385-395

26. Sadun AA, Bassi CH (1990) Optic nerve damage in Alzheimer's disease. Ophthalmology 97(1):9-17

27. Koronyo-Hamaoui M, Koronyo Y, Ljubimov AV et al (2011) Identification of amyloid plaques in retinas from Alzheimer's patients and noninvasive in vivo optical imaging of retinal plaques in a mouse model. NeuroImage 54(Suppl 1):S204-S217

28. Dehabadi MH, Davis BM, Wong TK, Cordeiro MF (2014) Retinal manifestations of Alzheimer's disease. Neurodegener Dis Manag 4(3):241-252

29. Guo L, Duggan J, Cordeiro MF (2010) Alzheimer's disease and retinal neurodegeneration. Curr Alzheimer Res 7:3-14
30. Williams PA, Thirgood RA, Oliphant $\mathrm{H}$ et al (2013) Retinal ganglion cell dendritic degeneration in a mouse model of Alzheimer's disease. Neurobiol Aging 34(7):1799-1806

31. Van Buren JM (1963) Trans-synaptic retrograde degeneration in the visual system of primates. J Neurol Neurosurg Psychiatry 26:402409

32. Beatty RM, Sadun AA, Smith L, Vonsattel JP, Richardson EP Jr (1982) Direct demonstration of transsynaptic degeneration in the human visual system: a comparison of retrograde and anterograde changes. J Neurol Neurosurg Psychiatry 45:143-146

33. Fletcher WA, Hoyt WF, Narahara MH (1988) Congenital quadrantanopia with occipital lobe ganglioglioma. Neurology 38 : 1892-1894

34. Jindahra P, Petrie A, Plant GT (2009) Retrograde trans-synaptic retinal ganglion cell loss identified by optical coherence tomography. Brain 132:628-634

35. Jindahra P, Petrie A, Plant GT (2012) The time course of retrograde trans-synaptic degeneration following occipital lobe damage in humans. Brain 135:534-541

36. Katsutoshi $\mathrm{G}$ et al (2016) Sectoral analysis of the retinal nerve fiber layer thinning and its association with visual field loss in homonymous hemianopia caused by post-geniculate lesions using spectraldomain optical coherence tomography. Graefes Arch Clin Exp Ophthalmol 254:745-756

37. Tsai CS, Ritch R, Schwartz B, Lee SS, Miller NR, CHI T et al (1991) Optic-nerve head and nerve-fiber layer in Alzheimer's disease. Arch Ophthalmol 109:199-204

38. Hedges TR, Perez Galves R, Speigelman D, Barbas NR, Pelo E, Yardley CJ (1996) Retinal nerve fiber layer abnormalities in Alzheimer's disease. Acta Ophthalmol Scand 74:271-275

39. Kromer R, Serbecic N, Hausner L, Froelich L \& Beutelspacher SC (2013) Comparison of visual evoked potentials and retinal nerve fiber layer thickness in Alzheimer's disease. Front Neurol 4

40. Lu Y, Li Z, Zhang X, Ming B, Jia J, Wang R et al (2010) Retinal nerve fiber layer structure abnormalities in early Alzheimer's disease: evidence in optic coherence tomography. Neurosci Lett 480: 69-72

41. Ong Y-L, Ong Y-T, Cheung CY et al (2014) Potential applications of spectral-domain optical coherence tomography (SD-OCT) in the study of Alzheimer's disease. Proceedings of Singapore Healthcare 23:1

42. Danesh-Meyer HV, Birch H, Ku JY et al (2006) Reduction of optic nerve fibers in patients with Alzheimer disease identified by laser imaging. Neurology 67:1852-1854

43. Parisi V, Restuccia R, Fattaposta F, Mina C, Bucci MG, Pierelli F (2001) Morphological and functional retinal impairment in Alzheimer's disease patients. Clin Neurophysiol 112:1860-1867

44. Iseri PK, Altinas O, Tokay T, Yuksel N (2006) Relationship between cognitive impairment and retinal morphological and visual functional abnormalities in Alzheimer disease. J Neuroophthalmol 26:18-24

45. Berisha F, Feke GT, Trempe CL, McMeel JW, Schepens CL (2007) Retinal abnormalities in early Alzheimer's disease. Invest Ophthalmol Vis Sci 48:2285-2289

46. Paquet C, Boissonnot M, Roger F, Dighiero P, Gil R, Hugon J (2007) Abnormal retinal thickness in patients with mild cognitive impairment and Alzheimer's disease. Neurosci Lett 420:97-9

47. Kesler A, Vakhapova V, Korczyn AD, Naftaliev F, Neudorfer M (2011) Retinal thickness in patients with mild cognitive impairment and Alzheimer's disease. Clin Neurol Neurosurg 113:523-526

48. Moschos MM, Markopoulos I, Chatziralli I, Vassilopoulos D et al (2012) Structural and functional impairment of the retina and optic nerve in Alzheimer's disease. Curr Alzheimer Res 9:782-788

49. Marziani E, Pomati S, Ramolofo P et al (2013) Evaluation of retinal nerve fiber layer and ganglion cell layer thickness in Alzheimer's 
disease using spectral-domain optical coherence tomography. Invest Ophthalmol Vis Sci 54:5953-5958

50. Ascaso FJ, Cruz N, Modrego PJ, Lopez-Anton R, Santabárbara J, Pascual LF et al (2014) Retinal alterations in mild cognitive impairment and Alzheimer's disease: an optical coherence tomography study. J Neurol 261:1522-1530. doi:10.1007/s00415-014-7374-z

51. Garcia-Martin ES, Rojas B, Ramirez AI, de Hoz R, Salazar JJ, Yubero R, Gil P, Triviño A, Ramirez JM (2014) Macular thickness as a potential biomarker of mild Alzheimer's disease. Ophthalmology 121(5):1149-1151-3

52. Salobrar-Garcia E, Hoyas I, Ramirez JM et al (2014) Analysis of retinal peripapillary segmentation in early Alzheimer's disease patients. BioMed Res Int 1-8

53. Gao LY, Liu Y, Li X, Bai Q, Liu P (2015) Abnormal retinal nerve fiber layer thickness and macula lutea in patients with mild cognitive impairment and Alzheimer's disease. Arch Gerontol Geriatr 60: 162-167

54. Cheung CYL, Ong YT, Hilal S et al (2015) Retinal ganglion cell analysis using high-definition optical coherence tomography in patients with mild cognitive impairment and Alzheimer's disease. J Alzheimers Dis 45(1):45-56

55. Zlokovic BV (2011) Neurovascular pathways to neurodegeneration in Alzheimer's disease and other disorders. Nat Rev Neurosci 12(12):723-738

56. Cheung CY, Ong YT, Ikram MK, Ong SY, Li X, Hilal S, Catindig JA, Venketasubramanian N, Yap P, Seow D, Chen CP, Wong TY (2014) Microvascular network alterations in the retina of patients with Alzheimer's disease. Alzheimers Dement 10:135-142

57. Cheung CY, Ong YT, Ikram MK, Chen C, Wong TY (2014) Retinal microvasculature in Alzheimer's disease. J Alzheimers Dis 42: S339-S352

58. Frost S, Kanagasingam Y, Sohrabi H, Vignarajan J, Bourgeat $\mathrm{P}$, Salvado O, Villemagne V, Rowe CC, Macaulay SL, Szoeke C, Ellis KA, Ames D, Masters CL, Rainey-Smith S, Martins RN, AIBL Research Group (2013) Retinal vascular biomarkers for early detection and monitoring of Alzheimer's disease. Transl Psychiatry 3:e233

59. Ramrattan RS, van der Schaft TL, MOoy CM, de Brujin WC, Mulder PG, de Jong PT (1994) Morphometric analysis of Bruch's membrane, the choriocapillaries, and the choroid in aging. Invest Ophthalmol Vis Sci 35(6):2857-2864

60. Mrejen S, Spaide RF (2013) Optical coherence tomography: imaging of the choroid and beyond. Surv Ophthalmol 58:387-429

61. Gharbiya M, Trebbastoni A, Parisi F, Campanelli A, Lena CD et al (2014) Choroidal thinning as a new finding in Alzheimer's disease: evidence from enhanced depth imaging spectral domain optical coherence tomography. J Alzheimers Dis 40:907-917

62. Bayhan HA, Aslan BS, Celikbilek A, Tanik N, Gurdal C (2015) Evaluation of the chorioretinal thickness changes in Alzheimer's disease using spectral-domain optical coherence tomography. Clin Exp Ophthalmol 43(2):145-151

63. Bulut M, Yaman A, Erol MK, Kurtuluș F, Toslak D, Doğan B, Kaya Başar E (2016) Choroidal thickness in patients with mild cognitive impairment and Alzheimer's type dementia. J Ophthalmol. doi:10.1153/2016/7291257

64. Kromer R, Serbecic N, Hausner L, Froelich L, Aboul-Enein F, Beutelspacher SC (2014) Detection of retinal nerve fiber layer defects in Alzheimer's disease using SD-OCT. Front Psychol 5:22

65. Larrosa JM, Garcia-Martin E, Bambo MP, Pinilla J, Polo V, Otin S, Satue M, Herrero R, Pablo LE (2014) Potential new diagnostic tool for Alzheimer's disease using a linear discriminant function for Fourier domain optical coherence tomography. Invest Ophthalmol Vis Sci 55(5):3043-3051
66. Polo V, Garcia-Martin E, Bambo MP, Pinilla J, Larrosa JM, Satue M, Otin S, Pablo LE (2014) Reliability and vailidity of Cirrus and Spectralis optical coherence tomography for detecting retinal atrophy in Alzheimer's disease. Eye 28:680-690

67. Keller J, Sánchez-Dalmau BF, Villoslada P (2014) Lesions in the posterior visual pathway promote trans-synaptic degeneration of retinal ganglion cells. PLoS One 9(5):e97444. doi:10.1371 /journal.pone.0097444

68. Herro AM, Lam BL (2015) Retrograde degeneration of retinal ganglion cells in homonymous hemianopsia. Clin Ophthalmol 9:10571064

69. Ong Y-T, Hilal S, Cheung CY, Venketasubramanian N, Niessen WJ, Vrooman H, Anuar AR, CHw M, Chen C, Wong TY, Ikram MK (2015) Retinal neurodegeneration on optical coherence tomography and cerebral atrophy. Neurosci Lett 584:12-16. doi:10.1016/j. neulet.2014.10.010

70. Kirbas S, TUrkyilmaz K, Anlar O, Furekci A, Durmus M (2013) Retinal nerve fiber layer thickness in patients with Alzheimer disease. J Neuroophthalmol 33(1):58-61

71. Bambo MP, Garcia-Martin E, Otin S, Pinilla J, Larrosa JM, Polo V, Pablo LE (2015) Visual function and retinal nerve fibre layer degeneration in patients with Alzheimer disease: correlations with severity of dementia. Acta Ophthalmol 93(6):e507-e508

72. Liu D, Zhang L, Li Z, Zhang X, Wu Y, Yang H, Min B, Zhang X, $\mathrm{Ma} \mathrm{D}, \mathrm{Lu}$ Y (2015) Thinner changes of the retinal nerve fiber layer in patients with mild cognitive impairment and Alzheimer's disease. BMC Neurol 15:14

73. He X-F, Liu Y-T, Peng C, Zhang F, Zhuang S, Zhang J-S (2012) Optical coherence tomography assessed retinal nerve fiber layer thickness in patients with Alzheimer's disease: a meta-analysis. Int J Ophthalmol 5(3):401-405

74. Thomson KL, Yeo JM, Waddell B, Cameron JR, Pal S (2015) A systematic review and meta-analysis of retinal nerve fiber layer change in dementia, using optical coherence tomography. Alzheimers Dement 1:136-143

75. Coppola G, Di Renzo A, Ziccardi L, Martelli F, Fadda A, Manni G et al (2015) Optical coherence tomography in Alzheimer's disease: a meta-analysis. PLoS ONE 10(8):e0134750. doi:10.1371/journal. pone. 0134750

76. Moreno-Ramos T, Benito-Leon J, Villarejo A, Bermejo-Pareja F (2013) Retinal nerve fiber layer thinning in dementia associated with Parkinson's disease, dementia with Lewy bodies, and Alzheimer's disease. J Alzheimers Dis 34:659-664

77. Oktem EO, Derle E, Kibaroglu S, Oktem C, Akkoyun I, Can U (2014) The relationship between the degree of cognitive impairment and retinal nerve fiber layer thickness. Neurol Sci 36(7): 1141-1146

78. Choi W, Mohler KJ, Potsaid B, Lu CD, Liu JJ et al (2013) Choriocapillaris and choroidal microvasculature imaging with ultrahigh speed OCT angiography. PLoS ONE 8(12):e81499. doi:10.1371/journal.pone.0081499

79. McKhann GM et al (2011) The diagnosis of dementia due to Alzheimer's disease: recommendations from the national institute on aging-Alzheimer's association workgroups on diagnostic guidelines for Alzheimer's disease. Alzheimers Dement 7:263-269. doi:10.1016/j.jalz.2011.03.005

80. Sperling RA et al (2011) Toward defining the preclinical stages of Alzheimer's disease: recommendations from the national institute on aging-Alzheimer's association workgroups on diagnostic guidelines for Alzheimer's disease. Alzheimers Dement 7:280-292. doi:10.1016/j.jalz.2011.03.003 